\title{
USO DA FUNÇÃO WEIBULL DE TRÊS PARÂMETROS EM UM MODELO DE DISTRIBUIÇÃO DIAMÉTRICA PARA PLANTIOS DE EUCALIPTO SUBMETIDOS A DESBASTE ${ }^{1}$
}

\author{
Daniel Henrique Breda Binoti ${ }^{2}$, Helio Garcia Leite ${ }^{3}$, Gilciano Saraiva Nogueira ${ }^{4}$, Mayra Luiza Marques \\ da Silva ${ }^{2}$, Silvana Lages Ribeiro Garcia ${ }^{5}$ e Jovane Pereira da Cruz ${ }^{6}$
}

\begin{abstract}
RESUMO - O objetivo deste estudo foi propor um modelo de distribuição diamétrica para povoamentos de eucalipto submetidos ao desbaste, com a inclusão do parâmetro de locação da função Weibull. Essa função foi ajustada a dados de 48 parcelas permanentes instaladas em um povoamento desbastado de um clone híbrido de eucalipto (Eucalyptus grandis x Eucalyptus urophylla), localizado na região Nordeste do Estado da Bahia, Brasil. A aderência foi avaliada pelo teste de Kolmogorov-Smirnorv (K-S). A redistribuição teórica dos diâmetros foi feita a partir de equações lineares e não lineares entre os parâmetros da função Weibull em uma idade futura e os parâmetros, em uma idade atual e associados a algumas características do povoamento em idades atual e futura. O sistema de equações gerado foi avaliado utilizando-se o coeficiente de determinação ajustado e o coeficiente de correlação entre frequências observadas e frequências estimadas e a análise gráfica dos resíduos. O sistema proposto resultou em estimativas precisas e consistentes de crescimento por classe de diâmetro.
\end{abstract}

Palavras-chave: Modelagem de crescimento, Eucalipto e Função densidade de probabilidade.

\section{THREE-PARAMETER WEIBULL DISTRIBUTION IN A DIAMETRIC DISTRIBUTION MODEL FOR THINNING EUCALIPTUS STANDS}

\begin{abstract}
The objective of the study was to propose a diametric distribution model for eucalyptus stands subjected to thinning, including the location parameter of the Weibull function. The Weibull function was adjusted to data of 48 permanent plots installed in a thinned stand of a hybrid clone of eucalyptus (Eucalyptus grandis $x$ Eucalyptus urophylla), located in the northeast area of the state of Bahia, Brazil. Adherence was evaluated by the Kolmogorov-Smirnorv test. The theoretic redistribution of diameters by class was made through equations that include both linear and non-linear relations between the Weibull function parameters in a future age and the parameters in the present age and with some characteristics of the stand in the present and future age. The equations were analyzed through the adjusted determination coefficient, correlation coefficient and graph analyses of the residues. This system generated accurate and consistent growth estimates per diameter class.
\end{abstract}

Keywords: Growth modeling, Eucalyptus and Probability Density Function.

\section{INTRODUÇÃO}

A previsão da distribuição de diâmetros em povoamentos florestais é de grande importância para o planejamento, permitindo efetuar a prognose de multiprodutos da madeira (RENNOLLS et al., 1985) e simular desbastes. A modelagem usualmente é feita em dois estágios: primeiro, uma função de densidade probabilidade (f.d.p.) é ajustada aos dados de distribuição de diâmetros de cada parcela e em cada medição; e, em seguida, os parâmetros da f.d.p. são correlacionados com atributos do povoamento (CAMPOS e LEITE, 2006).

\footnotetext{
${ }^{1}$ Recebido em 21.06.2008 e aceito para publicação em 14.10.2009.

${ }^{2}$ Programa de Pós-Graduação em Ciência Florestal, Universidade Federal de Viçosa - Viçosa, MG - Brasil..E-mail: <danielbinoti@yahoo.com.br>e <mayra_ufv@yahoo.com.br>.

${ }^{3}$ Departamento de Engenharia Floresta, Universidade Federal de Viçosa - Viçosa, MG - Brasil E-mail: <hgleite@ gmail.com>.

${ }^{4}$ Universidade Federal dos Vales do Jequitinhonha e Mucuri, UFVJM, Brasil. E-mail: <nogueirags@ yahoo.com.br>.

${ }^{5}$ Instituto Viçosence de Ensino e Pesquisa, Faculdade de Viçosa - FDV. E-mail: <garcias1@ yahoo.com.br〉.

${ }^{6}$ Bahia Pulp. E-mail: <jovane_cruz@bahiapulp.com>.
} 
A função densidade probabilidade Weibull é atualmente a mais utilizada no setor florestal. Em parte, isso ocorre devido ao fato de os plantios equiâneos apresentarem distribuições diamétricas assimétricas à direita, em razão de as árvores maiores apresentarem maiores taxas de crescimento que as menores (suprimidas). Isso gera uma configuração que coincide com as condições ideais para o ajuste dessa função (GUIMARÃES, 1994). Conforme Bailey e Dell (1973), ela pode ser expressa assim:

$f(X)=\left\{\frac{\gamma}{\beta}\left(\frac{(x-\alpha)}{\beta}\right)^{(\gamma-1)} e^{-\left(\frac{(x-\alpha)}{\beta}\right)^{\gamma}}\right.$,

para a $\leq \mathrm{x}<\infty$ em que $\alpha$ é o parâmetro de locação, $\beta$ é o parâmetro de escala $(\beta>0), \gamma$ o parâmetro de forma $(\beta>0)$ e $\gamma$ o centro de classe de diâmetro $(x \geq 0)$.

O ajuste da função Weibull pelo método da máxima verossimilhança proporciona estimativas mais confiáveis e com menor tendenciosidade do que outros métodos como o dos momentos e o dos percentis (BAILEY e DELL, 1973; CAMPOS e TURNBULL, 1981; FINGER, 1982; CAMPOS E LEITE, 2006).

O parâmetro de locação na maioria dos estudos sobre distribuição diamétrica tem sido considerado como o diâmetro mínimo do povoamento. Por isso, muitas vezes esse parâmetro é eliminado da função, assumindo o início desde a origem. Essa medida é tomada porque o parâmetro de locação apresenta baixa correlação com características do povoamento e sua exclusão contribui para maior facilidade de projeção dos parâmetros de forma e de escala (RUSTAGI, 1977; GUIMARÃES, 1994; BAILEY e DELL, 1973; NOGUEIRA, 2003). Poucos estudos foram feitos considerando-se o parâmetro de locação na projeção da distribuição diamétrica, porém a presença desse parâmetro aumenta a precisão do ajuste da função Weibull (BINOTI, 2008).
O objetivo deste estudo foi propor um modelo de distribuição diamétrica para povoamentos de eucalipto submetidos a desbastes seletivos, incluindo a projeção do parâmetro de locação da distribuição no modelo.

\section{MATERIALE MÉTODOS}

Foram utilizados dados de um experimento sobre desbastes, instalado em três povoamentos do híbrido Eucalyptus grandis x Eucalyptus urophylla, em uma empresa florestal localizada na região Nordeste do Estado da Bahia, Brasil. O experimento foi localizado em três instalações, abrangendo locais de capacidades produtivas extremas e intermediária. Em cada instalação foram aplicados quatro tratamentos em dois blocos. Cada bloco continha duas repetições, totalizando 48 parcelas, com área útil de $2.600 \mathrm{~m}^{2}$, sendo o espaçamento inicial entre as árvores de 3,0 x 3,0 m. Os tratamentos aplicados corresponderam a 20\%,35\% e 50\% de área basal removida em cada desbaste, eliminando-se os piores indivíduos e seguindo os princípios mencionados em Marshall e Curtis (2002). O quarto tratamento consistiu em um peso de $35 \%$ de desbaste acrescido de desrama artificial na idade de 1,5 ano. Informações da área onde se encontra o experimento são apresentadas no Tabela 1 .

As parcelas permanentes foram mensuradas aos 27, 40, 50, 58, 61, 76, 87, 101 e 112 meses, com um desbaste sendo realizado aos 58 meses. Em cada ocasião, foram medidos o diâmetro e a altura a 1,3 m de altura (dap) de todas as árvores da parcela, além das correspondentes alturas totais.

\subsection{Distribuição Diamétrica}

Ajustou-se em todas as parcelas a função Weibull com três parâmetros. Para os ajustes, os dados foram agrupados em classes com amplitude de $1,0 \mathrm{~cm}$. Utilizou-se em todas as funções um fator assintótico definido como o número de árvores da parcela.

Tabela 1 - Informações sobre o experimento.

Table 1 - Location of the experiment.

\begin{tabular}{|c|c|c|c|c|c|}
\hline Projeto & Município & $\begin{array}{l}\text { Latitude } \\
\text { (S) }\end{array}$ & $\begin{array}{l}\text { Longitude } \\
\text { (W) }\end{array}$ & $\begin{array}{l}\text { Altitude média } \\
\qquad(\mathrm{m})\end{array}$ & $\begin{array}{c}\text { Precipitação } \\
\text { média anual }(\mathrm{mm})\end{array}$ \\
\hline A & Inhambupe-BA & $11^{\circ} 52^{\prime}$ & $38^{\circ} 32^{\prime}$ & 285 & 900 \\
\hline B & Inhambupe-BA & $12^{\circ} 03^{\prime}$ & $38^{\circ} 28^{\prime}$ & 290 & 1.100 \\
\hline $\mathrm{C}$ & Esplanada-BA & $11^{\circ} 47^{\prime}$ & $37^{\circ} 55^{\prime}$ & 150 & 1.200 \\
\hline
\end{tabular}

R. Árvore, Viçosa-MG, v.34, n.1, p.147-156, 2010 
Em teoria, é possível calcular a probabilidade (L) dos valores específicos da variável dependente que ocorre na amostra, considerando-se o respectivo modelo de regressão. Todas as observações são valores independentes, resultando em uma soma geométrica das probabilidades de cada observação, tendo em conta os respectivos modelos e parâmetros para os valores de x. A soma geométrica resulta da multiplicação das probabilidades individuais em todos os casos. Também se pode expressar essa função como um logaritmo natural. Nesse caso, a soma geométrica torna-se uma soma aritmética regular. O ajuste da função foi realizado empregando-se o software kyplot versão 2.0 beta 15 (YOSHIOKA, 2001).

\subsection{Avaliação dos ajustes}

Para testar a aderência da função aos dados, utilizouse o teste Kolmogorov-Smirnorv (SOKAL e ROHLF, 1981; GIBBONS e SUBHABRATA, 1992). Esse teste compara a frequência acumulada estimada com a observada, sendo a classe de maior divergência a estatística do teste $(d n)$ :

$d n=M A X[F(X)-S(X)]$ em que $\mathrm{S}(\mathrm{X})$ é a frequência acumulada observada, e $\mathrm{F}(\mathrm{X})$ é a frequência estimada pela função-densidade probabilidade. $O$ teste foi aplicado a cada ajuste, a $1 \%$ de significância.

\subsection{Modelo de distribuição diamétrica}

O modelo de distribuição diamétrica utilizado neste trabalho foi construído com base no modelo proposto por Nogueira et al. (2005), sendo composto pelas seguintes relações funcionais:

$$
\begin{aligned}
& L n \beta_{2}=\operatorname{Ln} \beta_{1}\left(\frac{I_{1}}{I_{2}}\right)+a_{1}\left(1-\frac{I_{1}}{I_{2}}\right) d \max _{2} \\
& \alpha_{2}=\alpha_{1}\left(\frac{I_{1}}{I_{2}}\right)+a_{1}\left(1-\frac{I_{1}}{I_{2}}\right) d \min _{2} \\
& L n \gamma_{2}=\left(a_{1} \mathrm{e}^{\left(-\left(a_{2}\right)\left(I_{2}^{a_{3}}-I_{1}^{a_{3}}\right)\right)}\right)+a_{4} \beta_{2}+a_{5} L n \gamma_{1} \\
& d \max _{2}=d \max _{1}\left(\frac{I_{1}}{I_{2}}\right)+a_{1}\left(1-\frac{I_{1}}{I_{2}}\right) q_{1} \\
& N_{2}=N_{1} \mathrm{e}^{\left(-\left(a_{1}\right)\left(I_{2}^{a_{2}}-I_{1}^{a_{2}}\right)\right)}
\end{aligned}
$$

em que:

$I_{1}$ e $I_{2}$ são as idades atual e futura, respectivamente, em meses; $\gamma_{1}$ e $\gamma_{2}$ são os parâmetros de forma da função Weibull nas idades atual e futura; $\beta_{1}$ e $\beta_{2}$ são os parâmetros de escala dessa função nas idades atual e futura; $a_{1}$ e $a_{2}$ são os parâmetros de locação da função nas idades atual e futura; $\operatorname{dmax}_{1}$ e dmax $_{2}$ são os diâmetros máximos nas idades atual e futura, em cm, $N_{1}$ e $N_{2}$ são as frequências de árvores por hectare nas idades atual e futura; $q_{1}$ é o diâmetro médio; $L n$ indica o logaritmo neperiano; e $a_{1}, a_{2}, a_{3}$, $a_{4}$ e $a_{5}$ são os coeficientes das equações.

Após os ajustes da função Weibull de cada parcela em cada ano de medição, procedeu-se ao ajuste dos modelos pelo método iterativo Quasi-Newton, empregando o software Statistica 8 (STATSOFT Inc., 2009).

\subsection{Avaliação da redistribuição diamétrica}

A redistribuição de diâmetros foi avaliada com base na capacidade do sistema de equações em estimar o diâmetro máximo, o número total de árvores e os parâmetros $\gamma, \beta$ e $\alpha$ e recuperar a distribuição de diâmetros para qualquer idade. Essa avaliação foi feita com base no coeficiente de determinação ajustado para modelos lineares, no coeficiente de correlação entre os valores observados e estimados de frequência para modelos não lineares e na análise gráfica dos resíduos, para modelos lineares e não lineares.

Para avaliar a consistência, a capacidade de projeção e o realismo biológico do sistema, foram elaborados os seguintes gráficos da distribuição média de diâmetros: distribuição inicial observada com a projetada para o mesmo momento inicial; distribuição observada em idade futura com a projetada para a mesma idade futura, a partir de uma distribuição inicial; e distribuições projetadas para idades futuras a partir de diferentes distribuições iniciais.

\section{RESULTADOS}

Todos os ajustes da função Weibull realizados apresentaram aderência pelo teste de KolmogorovSmirnorv. Na Figura 1, apresenta-se graficamente o resultado do ajuste da função Weibull de algumas parcelas

R. Árvore, Viçosa-MG, v.34, n.1, p.147-156, 2010 
escolhidas aleatoriamente em cada uma das idades. $\mathrm{O}$ sistema de equações ajustado é apresentado na Tabela 2. Na Figura 2 são apresentados os gráficos de resíduos das equações desse sistema. Com as equações ajustadas, foram feitas simulações da distribuição de diâmetros em idades futuras (Figura 3).

Tabela 2 - Parâmetros estimados e coeficientes de determinação ajustado $\left(\bar{R}_{\hat{y y y}}^{2}\right)$ e de correlação $\left(R_{\hat{y y}}\right)$ das equações do modelo de distribuição diamétricas.

Table 2 - Estimated parameters and adjusted coefficients of determination $\left(\bar{R}_{\hat{y}}^{2}\right)$ and correlation $\left(R_{\hat{y} y}\right)$ for the equations of the diameter distribution model.

\begin{tabular}{|c|c|}
\hline $\operatorname{Ln} \gamma_{2}=\left(0,528292 \mathrm{e}^{\left(-(1189,357)\left(I_{2}^{-0,000098}-I_{1}^{-0,000028}\right)\right)}\right)+0,095207 \beta_{2}+0,060651 \operatorname{Ln} \gamma_{1}$ & $R_{\hat{y} y}=0,931$ \\
\hline$d \max _{2}=d \max _{1}\left(\frac{I_{1}}{I_{2}}\right)+2,038005\left(1-\frac{I_{1}}{I_{2}}\right) q_{1}$ & $\bar{R}_{\hat{y} y}^{2}=0,959$ \\
\hline $\operatorname{Ln} \beta_{2}=\operatorname{Ln} \beta_{1}\left(\frac{I_{1}}{I_{2}}\right)+0,192178\left(1-\frac{I_{1}}{I_{2}}\right) d \max _{2}$ & $\bar{R}_{\hat{y} y}^{2}=0,700$ \\
\hline$\alpha_{2}=\alpha_{1}\left(\frac{I_{1}}{I_{2}}\right)+0,039135\left(1-\frac{I_{1}}{I_{2}}\right) d \min _{2}$ & $\bar{R}_{\hat{y y}}^{2}=0,817$ \\
\hline$N_{2}=N_{1} \mathrm{e}^{\left(-(0,000007)\left(I_{2}^{1,439667}-I_{1}^{1,483067}\right)\right)}$ & $R_{\hat{y y y}}=0,993$ \\
\hline
\end{tabular}

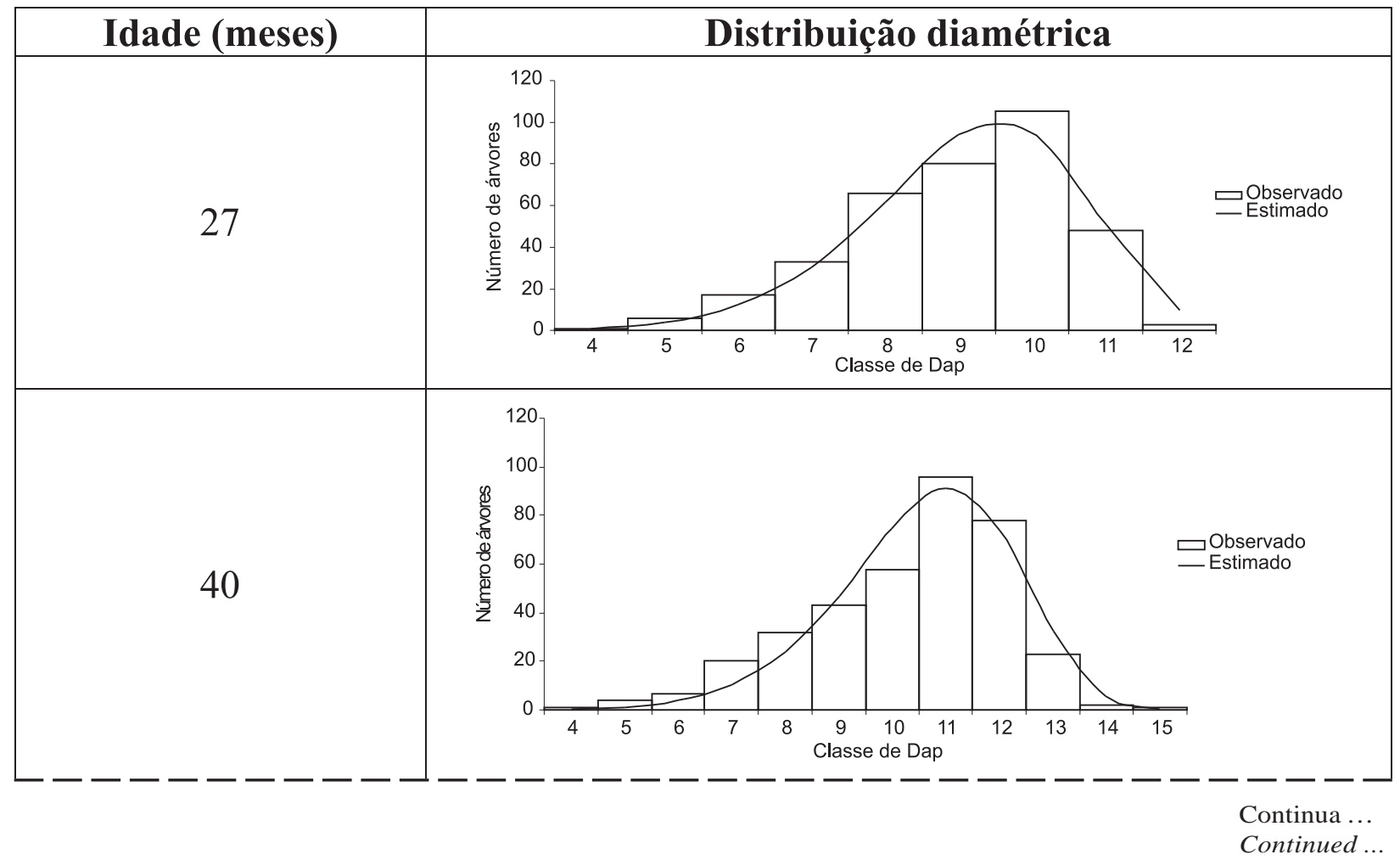

R. Árvore, Viçosa-MG, v.34, n.1, p.147-156, 2010 
Figura 1 - Cont.

Figura 1 - Cont.

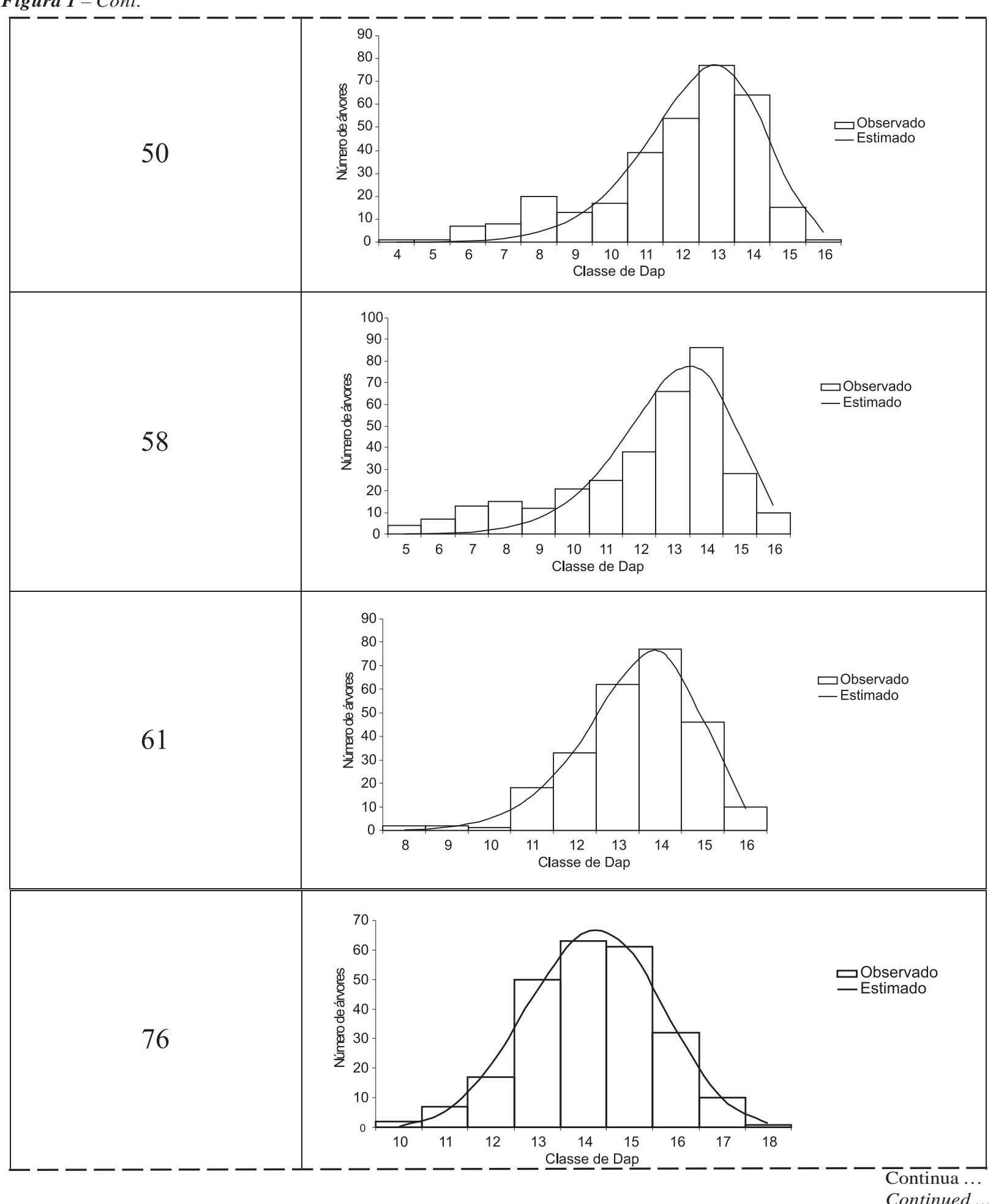


Figura 1 - Cont.

Figura 1 - Cont.

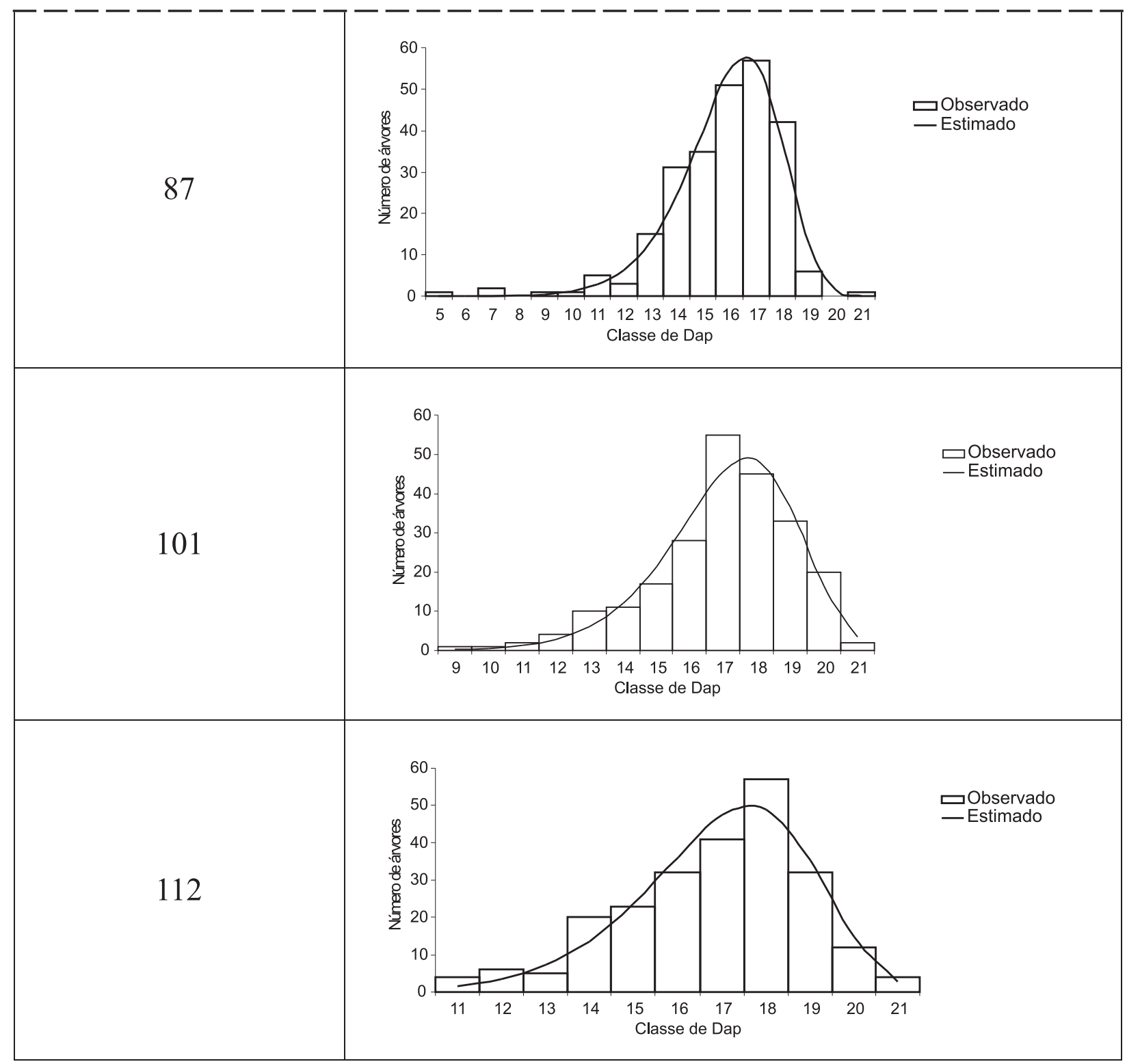

Figura 1 - Valores de distribuição diamétrica observados e estimados pela função Weibull de nove parcelas selecionadas ao acaso em cada idade.

Figure 1 - Observed and estimated frequency diametric distribution for the Weibull function of nine plots randomly selected in each age.

R. Árvore, Viçosa-MG, v.34, n.1, p.147-156, 2010 


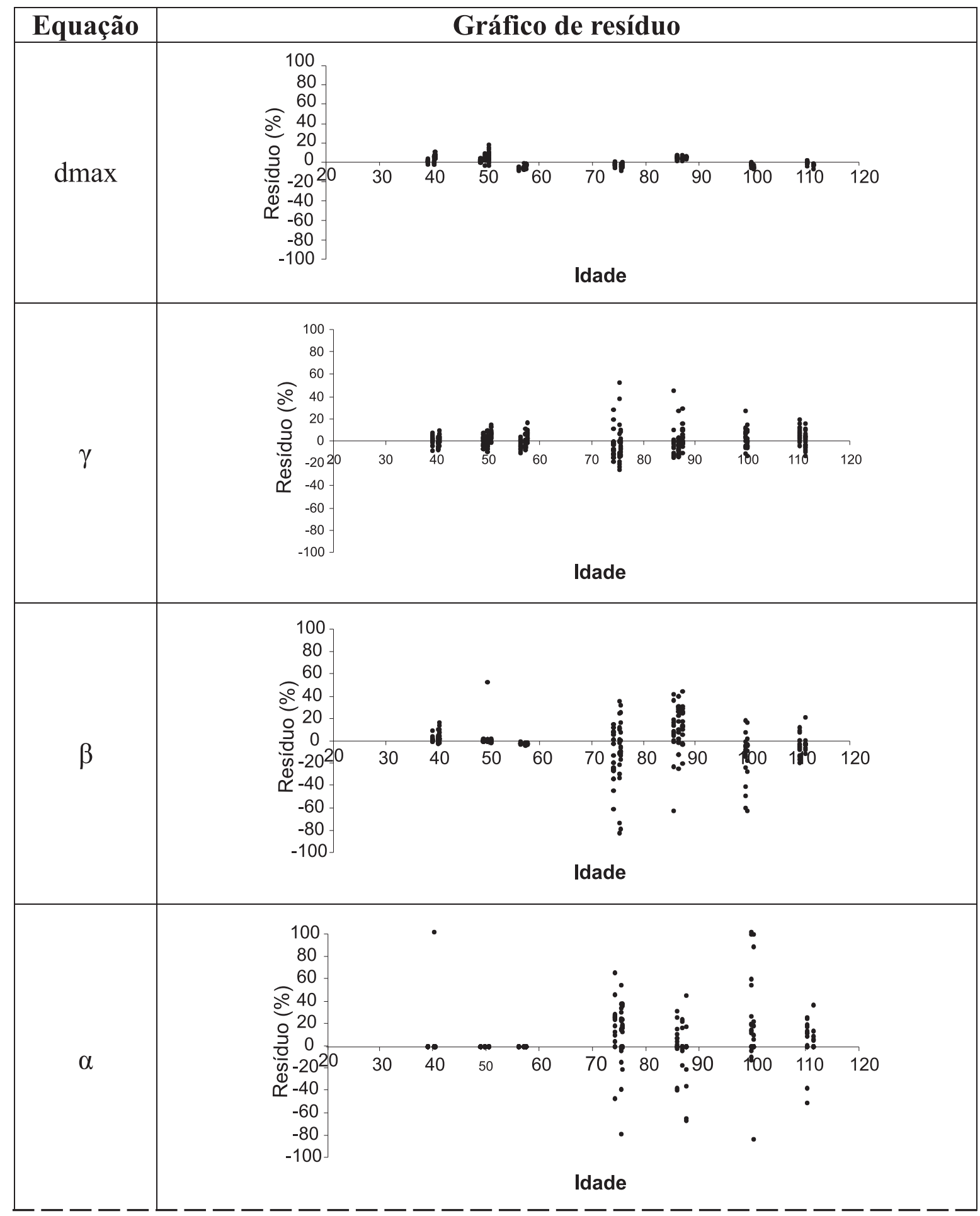


Figura 2 - Cont.

Figura 2 - Cont.

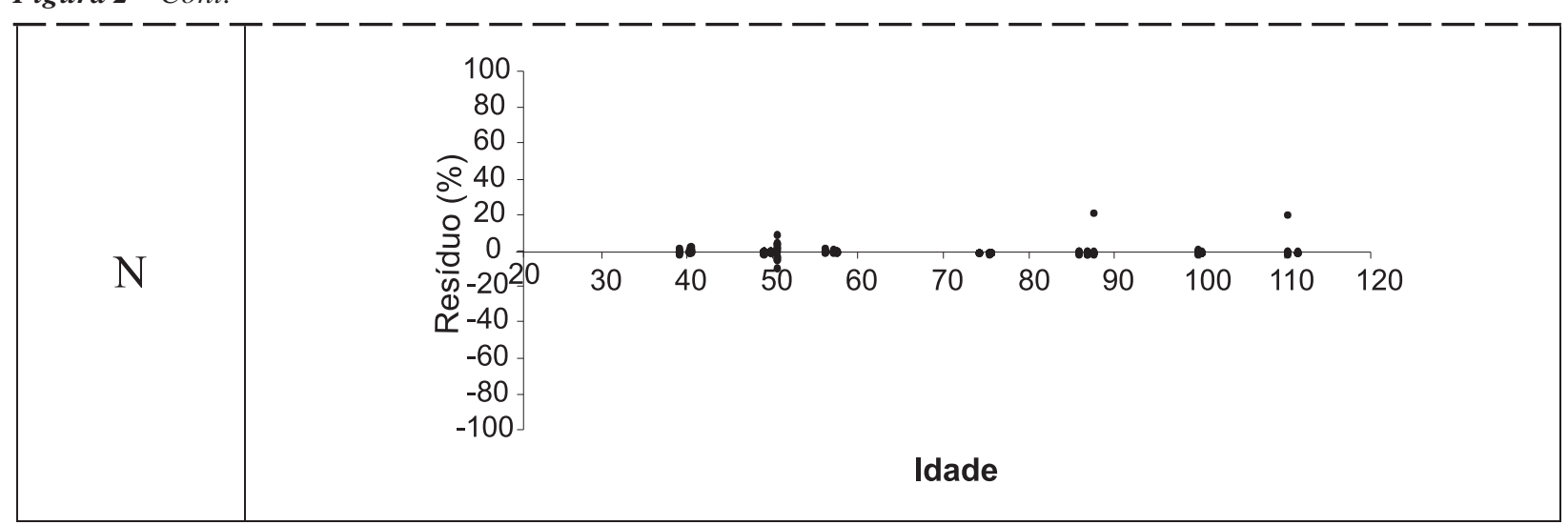

Figura 2 - Distribuição dos resíduos percentuais entre os valores observados e recuperados pelas equações que compõem o modelo de distribuição de diâmetro.

Figure 2 - Distribution of percent deviations between observed and recovered values by the equations that compose the diameter distribution model.

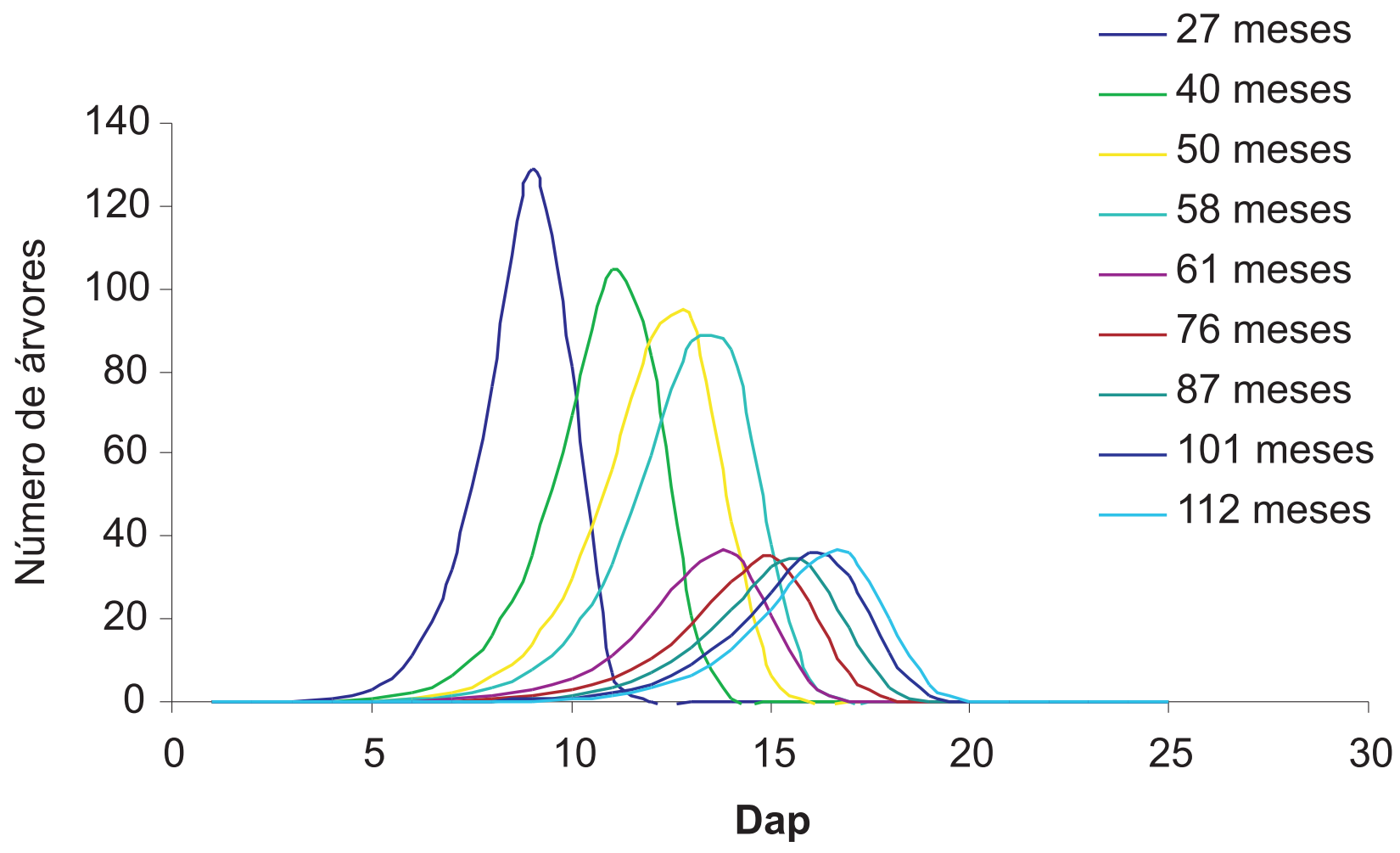

Figura 3 - Projeção da distribuição diamétrica para idades futuras.

Figure 3 - Projection of the diametric distribution for future ages.

R. Árvore, Viçosa-MG, v.34, n.1, p.147-156, 2010 


\section{DISCUSSÃO}

Todos os ajustes da função Weibull resultaram em aderência pelo teste de Kolmogorov-Smirnorv ( $p>0,01$ ). Isso comprova a flexibilidade dessa função, que apresenta pontos de inflexão variando desde zero até 0,63 (GUIMARÃES, 1994).

Na Tabela 1, verifica-se que as equações ajustadas resultaram em valores relativamente altos de coeficiente de correlação e de determinação. Algumas equações (Tabela 1) não são consistentes, ou seja, ao estimar a variável de uma mesma idade resulta em valores diferentes daqueles observados. Porém, as variáveis incluídas na equação melhoraram a qualidade do ajuste; além disso, modelos de crescimento e produção são aplicados, principalmente, para promover projeções em idades futuras e, raramente, para idade atual (LOPES, 2007).

No parâmetro de locação ocorreram maiores dispersões de resíduos após o desbaste. Mesmo assim, o modelo apresentado é mais consistente que os modelos e métodos usuais de estimação desse parâmetro, em que são utilizadas regressões lineares (KNOBELL, 1986; NOGUEIRA et al., 2005).

O realismo biológico do sistema de equações pode ser comprovado na Figura 3. Verifica-se, nessa figura, a estagnação da distribuição de diâmetros na idade imediatamente antes do desbaste, quando o número de árvores que ingressaram em novas classes foi reduzido. Com o desbaste, a competição entre as árvores foi reduzida, permitindo incremento maior de indivíduos em novas classes (GARCIA, 2000; CAMPOS e LEITE, 2006).

\section{CONCLUSÃO}

O modelo apresentado neste estudo é consistente e compatível, sendo indicado para uso em manejo de florestas de eucalipto submetidas a desbaste.

\section{REFERÊNCIAS}

BAILEY, R.; DELL, T. Quantifying diameter distributions with the Weibull function. Forest Science. v.19, n.2.p.97-104, 1973.

BINOTI, D. H. B. Funções densidade de probabilidade para a descrição da distribuição diamétrica de povoamentos desbastados de Tectona grandis. 2008. 42f. Monografia (Graduação em Engenharia Florestal) - Universidade Federal de Viçosa, Viçosa, MG, 2008.
CAMPOS, J. C. C.; LEITE, H. G. Mensuração florestal: perguntas e respostas. 2.ed. Viçosa, MG: Universidade Federal de Viçosa, 2006. 470p.

CAMPOS, J. C. C.; TURNBULL, K. Um sistema para estimar a produção por classe de diâmetro e sua aplicação na interpretação do efeito de desbaste. Árvore, v.5, n.1, p.1-16, 1981.

FINGER, C. A. G. Distribuição de diâmetros em Acácia negra Acacia mearnsii de Wild, em diferentes povoamentos e idades. 1982. $129 \mathrm{f}$. Dissertação (Mestrado Engenharia Florestal) Universidade Federal do Paraná, Curitiba, 1982.

GARCIA, S. L. R. Ingresso percentual: teoria e aplicações em ciência florestal. Viçosa, MG: 2000. 20p. (Não Publicado).

GIBBONS, J. D.; SUBHABRATA, C. Nonparametric statistical inference. 3-ed. New York: Marcel Dekker, 1992. 544p. (Statistics: textbook and monograph, v.31).

GUimarÃES, D. P. Desenvolvimento de um modelo de distribuição diamétrica de passo invariante para prognose e projeção da estrutura de povoamentos de eucalipto. 1994. 178f. Tese (Doutorado em Ciência Florestal) - Universidade Federal de Viçosa, Viçosa, MG, 1994.

KNOEBELL, B. R.; BURKHART, H. E.; BECK, D. E. A growth and yield model for thinned stands of yellow-poplar. Forest Science, v.32, n.2, p.162, 1986. (Monograph, 27)

MARSHALL, D. D.; CURTIS, R. O. Levels-ofgrowing-stock cooperative study in Douglas-fir. Victoria: USDA, 2002.4p. (Information Forestry).

LOPES, P. F. Modelo de distribuição de diâmetros para clones de eucalipto em sistema agroflorestal. 2007. 32f. Tese (Mestrado em Ciência Florestal) - Universidade Federal de Viçosa, Viçosa,MG, 2007.

NOGUEIRA, G. S. Modelagem do crescimento e da produção de povoamentos de Eucalyptus sp. e de Tectona grandis submetidos a desbaste. 2003. 132f. Tese (Doutorado em Ciência Florestal) Universidade Federal de Viçosa, Viçosa, MG, 2003.

R. Árvore, Viçosa-MG, v.34, n.1, p.147-156, 2010 
NOGUEIRA, G. S. et al. Modelo de distribuição diamétrica para povoamentos de Eucalyptus sp. submetidos a desbaste. Árvore, v.29, n.4, p.579589, 2005.

RENNOLLS, K.; GEARY, D. N.; ROLLINSON, T. J. D. Characterizing diameter distributions by the use of the Weibull distribution. Forestry, v.58, n.1, p.57-66, 1985.

SOKAL, R. R.; ROHLF, F. J. Biometry. San Francisco: Freeman, 1981.859p.
RUSTAGI, K. P. Predicting stand structure in evenaged stands. In: GROWTH MODELS FOR LONG TERM FORECASTING OF TIMBER YIELDS, 1977, Blacksburg. Proceedings... [S.1.]: IUFRO, [1977]. p.1-16.

STATSOFT, Inc. STATISTICA (data analysis software system), version 8 . 2009. (Software Estatístico).

YOSHIOKA, Y. KyPlot Software. v2.00 beta 15. 2001 (Software Estatístico). 